\title{
Evaluation of placental function using near infrared spectroscopy during fetal growth restriction
}

\author{
Junichi Hasegawa*, Masamitsu Nakamura, Ryu \\ Matsuoka, Takashi Mimura, Kiyotake Ichizuka, \\ Akihiko Sekizawa and Takashi Okai
}

Department of Obstetrics and Gynecology, Showa

University School of Medicine, Tokyo, Japan

\section{Introduction}

Although various factors effect fetal growth restriction (FGR), its actual cause is rarely diagnosed prenatally. Placental morphological abnormalities and some umbilical cord abnormalities may be prenatally detected using ultrasonography. However, it is still difficult to evaluate placental function and its relationship to FGR, though the morphological alterations of the placenta can be evaluated retrospectively by pathological examinations. Antenatally, only some circulatory activity can be measured in the uterus but no direct methods have yet been established to determine the functional status of the placenta. If the placental oxygenation conditions could be non-invasively measured during pregnancy, it would provide valuable information about oxygenation of the fetus, association between growth restriction, hypoxia, etc.

Near-infrared spectroscopy (NIRS), first described by Jobsis in 1977 [2], is a non-invasive and convenient method of monitoring tissue oxygenation of a number of intact organs, especially in neurology and cardiology. However, only a few studies [3,5] have applied NIRS for placental function.

The aim of this study was to investigate whether NIRS via the maternal abdominal approach is useful for evaluation of the placental function in FGR cases.

\section{Materials and methods}

A prospective cohort study was conducted at the Perinatal Center of Showa University School of Medicine between June 2007 and August 2008. A total of 326 pregnant Japanese women who planned to deliver at that hospital and whose placenta was located on the anterior wall or the fundus of the uterus were enrolled. Informed consent was obtained from all participants.

Transabdominal NIRS was conducted with the Hamamatsu NIRO 120 system (Hamamatsu Photonics KK, Hamamatsu, Japan) at around 20 weeks (18-22 weeks), 30 weeks (28-32 weeks) and after 36 weeks' gestation. The components of the NIRO system were described elsewhere [3]. Briefly, four pulsed laser diodes are used as the source of NIR light. These diodes typically produce light at wavelengths of $775,825,850$, and $905 \mathrm{~nm}$. NIR light is directed to the tissue via optical fibers in the probe. The light emerging from the tissue is returned to the instrument through another optical fiber in the probe and is detected by a photomultiplier tube. Transmitted light intensities are recorded. Based on these records, the changes in the concentration of the chromophores oxyhemoglobin $\left(\mathrm{HbO}_{2}\right)$ and $\mathrm{Hb}$ were calculated and displayed on the front panel of the instrument.

\author{
*Corresponding author: \\ Dr. Junichi Hasegawa \\ Department of Obstetrics and Gynecology \\ 1-5-8 Hatanodai \\ Shinagawa-ku \\ Tokyo \\ $142-8666$ \\ Japan \\ Tel.: +81-3-3784-8551 \\ Fax: +81-3-3784-8355 \\ E-mail: hasejun@oak.dti.ne.jp
}


Table 1 Demographics of pregnancies with AGA and SGA fetuses.

\begin{tabular}{llll}
\hline Groups & AGA & SGA & P-value \\
\hline Number of cases & 282 & 44 & $\mathrm{~ns}$ \\
Maternal age & $32.4 \pm 4.4$ & $31.4 \pm 4.4$ & $\mathrm{~ns}$ \\
Body mass index & $20.3 \pm 2.6$ & $20.1 \pm 2.3$ & $\mathrm{~ns}$ \\
Parity (median, range) & $0,0-7$ & $0,0-4$ & $<0.01$ \\
Gestation of age at delivery (w) & $38.8 \pm 1.6$ & $36.2 \pm 3.6$ & $<0.01$ \\
Neonatal birth weight (g) & $3027 \pm 356$ & $1986 \pm 647$ & $<0.1-10$ \\
Apgar 5 min (median, range) & $9,1-10$ & $9,2-10$ & $<0.01$ \\
Apgar 5 min (median, range) & $10,3-10$ & $7.31 \pm 0.07$ & $\mathrm{~ns}$ \\
Umbilical artery pH & $7.32 \pm 0.08$ & & \\
\hline
\end{tabular}

Data indicated means \pm SDs. Statistical analyses were performed between AGA and SGA by Mann-Whitney $U$-test.

$\mathrm{AGA}=$ appropriate for gestational age, $\mathrm{SGA}=$ small for gestational age, $\mathrm{SD}=$ standard deviation.

The NIRO-120 is based on the spatially-resolved spectroscopy approach and provides relative concentration changes (expressed in $\Delta \mu \mathrm{M})$ of $\mathrm{HbO}_{2}$, deoxyhemoglobin $(\mathrm{HHb})$, and the derived total hemoglobin content $\left(\mathrm{tHb}=\mathrm{HbO}_{2}+\mathrm{HHb}\right)$, while $\mathrm{HbO}_{2}$ and $\mathrm{HHb}$ concentration changes are calculated. Consequently, tissue oxygen indexes (TOIs) were calculated $\left(\mathrm{TOI}=\mathrm{HbO}_{2} / \mathrm{tHb}\right)[10]$.

Transmitting and receiving transducers were placed on the maternal abdomen just above the placenta with a 4-cm interval to obtain signals for the calculation of TOI. Subjects whose distance measured by ultrasonography between skin and placenta was more than $3 \mathrm{~cm}$, were not included in this study, because this fat may disturb obtaining accurate signals.

After delivery, the neonates were categorized into small for gestational age (SGA) or appropriate gestational age (AGA). SGA was defined as birth weight less than the $10^{\text {th }}$ percentile for gestational age referred to standard values of Japanese neonates. The maternal and fetal complications, prenatal ultrasound findings, and abnormalities of placental and umbilical cord were reviewed. Thereafter, the cases of SGA were estimated and stratified by the potential cause of growth restriction. The causes of growth restriction included the followings: maternal complication (heart abnormality, bronchial asthma, diabetes), uterine abnormality (bicornuate uterus, myoma), low body mass index $(<18)$, major fetal anomaly (heart abnormality, pyelectasis), smoking, umbilical cord abnormality (velamentous cord insertion, hypercoiled cord), placental abnormality (infarction, deformed placenta, lobed placenta) and severe preeclampsia (blood pressure $>160 \mathrm{~mm} \mathrm{Hg}$ or proteinuria $>2 \mathrm{~g} /$ day).

This study analyzed the relationship between gestational age at the time of measurement and TOIs, and that between TOIs and causes of FGR.

The data were entered into a computerized data analysis program (StatView for Windows, SAS Institute, Inc, Cary, NC). Continuous variables were compared using the Mann-Whitney $U$-test. The correlations between TOIs and the SD of neonatal birth weight were compared using Pearson's correlation coefficient. Multiple comparisons were used by an analysis of variance and Bonferroni post-hoc test. Statistical significance was defined as a P-value of $<0.05$.

\section{Results}

Among the total 326 cases, 44 (13.5\%) were SGA. The demographics of all pregnancies are shown in Table 1. Although there were no differences in maternal age, BMI, parity and umbilical artery $\mathrm{pH}$ between the groups, significant differences were observed in neonatal birth weight, in gestational age at delivery and in the Apgar score. The causes of growth restriction were stratified by maternal factors (bicornate uterus or small body mass index), umbilical cord abnormalities (hypercoiled cord or velamentous cord insertion), placenta abnormalities (infarction, lobed placenta or deformed placenta), pre-eclampsia (severe proteinuria and/or hypertension) and unexplained FGR.

TOI values $(\% \pm \mathrm{SD})$ in all cases were $76.6 \pm 4.2$, $74.0 \pm 4.7,72.7 \pm 4.8$ at 20,30 and after 36 weeks of gestation, respectively (Figure 1). TOI values decreased with gestational age, and the value were significantly lower at 30 and after 36 weeks than at 20 weeks of gestation $(\mathrm{P}<0.001)$.

No correlation was observed between the SD of the neonatal birth weight and the TOI measured immediately before delivery; (Figure 2), and regardless of the causes of FGR. The last values of TOIs before delivery were significantly higher in the AGA groups with uterine abnormalities, in the SGA groups with placental abnormalities and with pre-

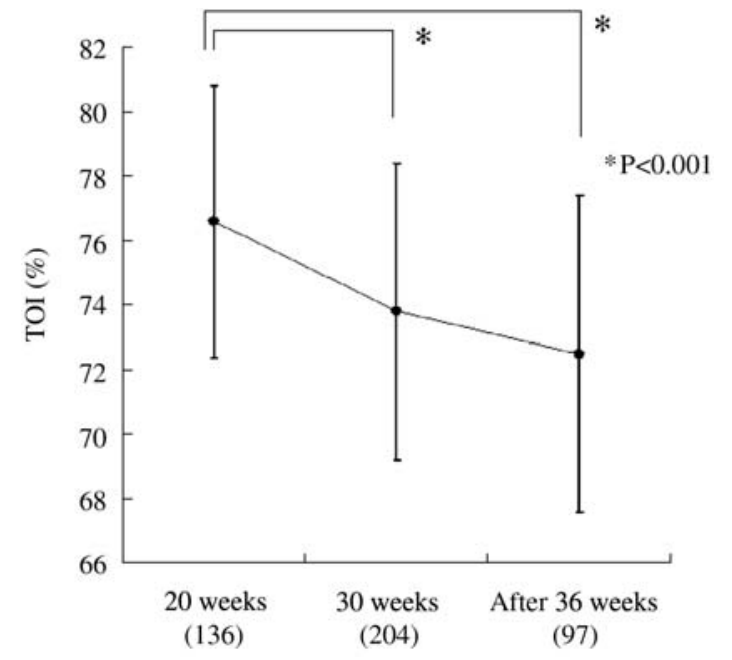

Figure 1 TOI values at 20 weeks, 30 weeks and after 36 weeks of gestation: TOI values were 76.6 $\pm 4.2,74.0 \pm 4.7,72.7 \pm 4.8$ $(\% \pm \mathrm{SD})$ at 20 weeks, 30 weeks and after 36 weeks of gestation, respectively. Bars indicate the mean values of TOIs and SDs. Statistical analyses were performed using ANOVA and the Bonferroni post-hoc test. 


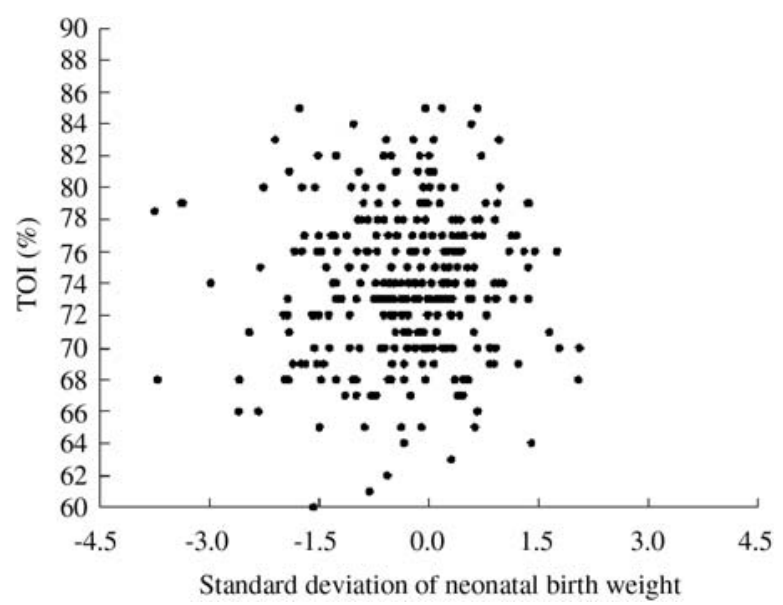

Figure 2 Correlation between the Z-score of neonatal birth weight and the last value of TOI before delivery: No correlation was observed between the Z-score of neonatal birth weight and last value of TOI before delivery.

eclampsia, and in all cases with pre-eclampsia compared to cases without (Table 2).

The last values of TOIs before delivery stratified by presumed main cause of SGA are shown in Table 3. TOIs in the SGA group with maternal complications were not different from the AGA group. The TOIs in SGA fetuses with severe pre-eclampsia and placental abnormalities were significantly higher than in the AGA group $(\mathrm{P}=0.002$ and 0.043). TOIs in the SGA group with umbilical cord abnormalities were significantly lower than in the AGA group $(\mathrm{P}=0.024)$.

\section{Discussion}

The main sources of change in the returning light signal by absorbing the specific wave length light were probably due to hemoglobin in the intervillous space, because it accounts for the majority of hemoglobin existing in the light pathway. Therefore, TOI mainly reflects the oxygenation of the intervillous blood.

A disturbance of blood flow in the placenta directly influences fetal growth. Kawamura et al. [5] reported an evaluation of the placental function using NIRS during pregnancy of 15 growth restricted fetuses compared with 15 normal pregnant women. TOI values of the FGR group were significantly higher than those of the controls (77.8 \pm 1.6 vs. $70.3 \pm 0.4)$. The authors maintained that oxygen exchange from maternal to fetal blood in the intervillous space may be impaired due to dysfunctional villi; as a result, there would be an increase in oxygen concentration in the intervillous spaces, thus yielding higher TOI values. Our results, however, have shown that TOIs in SGA vary according to the

Table 2 Relation between various maternal and intrauterine factors and tissue oxygen index (n) in 326 cases.

\begin{tabular}{|c|c|c|c|c|c|c|}
\hline \multirow[t]{2}{*}{ Factor } & \multicolumn{2}{|l|}{ AGA } & \multicolumn{2}{|l|}{ SGA } & \multicolumn{2}{|l|}{ Total } \\
\hline & No & Yes & No & Yes & No & Yes \\
\hline Maternal complication & $73.9 \pm 4.5(273)$ & $75.0 \pm 4.8(9)$ & $74.0 \pm 5.4(40)$ & $73.9 \pm 10.1(4)$ & $73.9 \pm 4.7(313)$ & $74.7 \pm 6.2(13)$ \\
\hline Uterine abnormality & $73.9 \pm 4.5(274)$ & $77.1 \pm 3.6 *(8)$ & $74.0 \pm 5.9(42)$ & $74.0 \pm 4.2(2)$ & $73.9 \pm 4.7(316)$ & $76.5 \pm 3.7(10)$ \\
\hline Low body mass index & $73.8 \pm 4.5(239)$ & $74.5 \pm 4.8(43)$ & $74.4 \pm 5.5(37)$ & $71.5 \pm 7.6(7)$ & $74.3 \pm 4.6(276)$ & $73.2 \pm 5.0(50)$ \\
\hline Fetal anomaly & $74.0 \pm 4.6(278)$ & $73.5 \pm 1.8$ & $74.0 \pm 5.8$ & - & $74.0 \pm 4.8(322)$ & $73.5 \pm 1.7(4)$ \\
\hline Smoking & $74.1 \pm 4.6(259)$ & $74.6 \pm 3.4(23)$ & $75.1 \pm 6.1(44)$ & - & $74.1 \pm 4.8(303)$ & $74.6 \pm 3.4(23)$ \\
\hline Umbilical cord abnormality & $73.9 \pm 4.6(256)$ & $75.2 \pm 4.3(26)$ & $73.9 \pm 5.0(31)$ & $74.1 \pm 7.6(13)$ & $73.9 \pm 4.6(287)$ & $74.8 \pm 5.5(39)$ \\
\hline Placental abnormality & $74.0 \pm 4.5(265)$ & $73.6 \pm 5.2(17)$ & $72.8 \pm 5.4(31)$ & $76.9 \pm 5.8 *(13)$ & $74.0 \pm 4.7(296)$ & $75.1 \pm 5.6(30)$ \\
\hline Pre-eclampsia & $74.0 \pm 4.5(280)$ & $78.0 \pm 9.0(2)$ & $73.2 \pm 5.6(38)$ & $79.4 \pm 4.1 *(6)$ & $73.9 \pm 4.6(318)$ & $79.1 \pm 5.1^{a}(8)$ \\
\hline
\end{tabular}

Data include multiplicities of abnormalities and indicate means \pm SDs. Statistical analyses were performed between no and yes in each section by Mann-Whitney $U$-test. a Significant difference between no and yes in the section $(\mathrm{P}<0.05)$.

$\mathrm{AGA}=$ appropriate for gestational age, $\mathrm{SGA}=$ small for gestational age, $\mathrm{SD}=$ standard deviation.

Table 3 Last TOI in AGA and SGA fetuses stratified by main causations of fetal growth restriction.

\begin{tabular}{lclc}
\hline Factor & Cases & TOI \pm SD (\%) & P-value \\
\hline AGA & 282 & $74.0 \pm 4.5$ & $74.0 \pm 5.8$ \\
SGA & 44 & $71.9 \pm 4.5$ & $0.203^{\mathrm{c}}$ \\
$\quad$ Maternal complication & 8 & $69.7 \pm 7.7$ & $0.024^{\mathrm{a}, \mathrm{b}}$ \\
Umbilical cord abnormality & 6 & $78.2 \pm 3.6$ & $0.043^{\mathrm{b}}$ \\
Placenta abnormalities & 5 & $\mathbf{7 9 . 2} \pm \mathbf{3 . 8}$ & $\mathbf{0 . 0 0 3}^{\mathrm{a}, \mathrm{c}}$ \\
Pre-eclampsia & 7 & $73.2 \pm 5.0$ & 0.498 \\
Unexplained factor & 18 & \\
\hline
\end{tabular}

Multiple comparisons were performed by analysis of variance $(\mathrm{P}=0.001)$ and Bonferroni post-hoc test. P-values were compared with TOIs in total cases of appropriate for gestational age. ${ }^{\mathrm{a}, \mathrm{b}, \mathrm{c}}$ Significant differences between two group.

$\mathrm{AGA}=$ appropriate for gestational age, $\mathrm{SGA}=$ small for gestational age, $\mathrm{TOI}=$ tissue oxygen index, $\mathrm{SD}=$ standard deviation . 
different causes. SGA due to pre-eclampsia and placental abnormalities had high TOIs, whereas those with umbilical cord abnormalities showed low TOIs. Then, when we compared TOIs between AGA and all SGA cases, there was no difference.

Kawamura et al. [5] suggested that high TOI values reflect high concentrations of $\mathrm{HbO}_{2}$ in intervillous space due to low maternal-fetal oxygen exchange through the villous membrane. In the current study, TOIs were high in, but not only cases with FGR due to severe pre-eclampsia, TOIs in placental abnormalities without pre-eclampsia were also higher than in controls. We suggest that the decrease of physical activity would most likely occur once the ability of the system to maintain normal status by increasing oxygen extraction and carrying capacity had been fully utilized. Perhaps, such procedures would preserve fetal growth during the initial decrease of oxygen supply. After this, the fetus would finally become growth restricted and in this situation, intravillous oxygen would be more than normal because less oxygen transportation to the fetus is caused by various placental abnormalities. Kaufmann et al. [4] suggested that preeclampsia is associated with generalized impairment of trophoblast invasion. Prefumo et al. [7] also suggested the poor development of the placenta in early stages of pregnancy may cause pre-eclampsia. Redman and Sargent [9] discussed that poor placentation does not always cause overt pre-eclampsia, but can be associated with a SGA fetuses. These cases might have a similar pathophysiological background as pre-eclampsia.

Interestingly, in the present study, the TOIs were significantly lower in the SGA cases due to umbilical cord abnormalities. There have been some reports on the pathophysiology of FGR with umbilical cord abnormalities. In a review by Benirschke [1], an association of chorangiosis with cord problems was noted. Ogino and Redline [6] postulated that the pathogenesis of chorangiosis was hypercapillarization related to an increased intramural pressure, perhaps related to venous obstruction at the umbilical cord or fetal cardiac level. These structural changes may promote oxygen transport from maternal to fetal circulation, then decrease TOI, which represent oxygen content in the intravillous space. Although no pathological examinations were done in the present study, a previous report [8] suggests that umbilical obstructive abnormalities cause the terminal villi to exaggerate the perfusion of oxygen between capillaries and the intervillous space. Consequently, oxygen might easily escape from the intervillous space due to villous complications, lowering the oxygenated hemoglobin in the intervillous space. Though it is difficult to explain why fetal growth is restricted in this circumstance, it is worth noting that the TOI were significantly lower in the growth restricted fetuses with umbilical cord abnormalities.

There are some limitations in measurement of oxygenation in the intervillous space of the placenta using NIRS. First, it is only available for cases with anterior placentas and with thin subcutaneous fat because light from the NIRS can only penetrate as far as $4-5 \mathrm{~cm}$. Second, the effects of other fac- tors in the light pathway, such as blood flow in the maternal abdominal wall and uterine wall, uterine contractions and intra-villous fetal blood flow, are unknown. Finally, the ranges of the TOIs were too narrow to identify the abnormal cases. However, despite the present limited applications of NIRS, it might be improved in the near future due to continuing advances in spectroscopy technology.

\section{Acknowledgements}

This study was supported in part by Grants-in-Aid for Scientific Research from the Ministry of Education, Science, Sport and Culture of Japan (No. 80365775). We wish to thank Prof. Naohiro Kanayama and Dr. Jun Kakogawa (Department of Obstetrics and Gynecology, Hamamatsu University School of Medicine, Japan) for their excellent advice. We are also grateful to the Hamamatsu Photonics KK (Hamamatsu, Japan) for providing the Hamamatsu NIRO 120 system.

\section{References}

[1] Benirschke K. Recent trends in chorangiomas, especially those of multiple and recurrent chorangiomas. Pediatr Dev Pathol. 1999;2:264-9.

[2] Jobsis FF. Noninvasive, infrared monitoring of cerebral and myocardial oxygen sufficiency and circulatory parameters. Science (New York, NY). 1977;198:1264-7.

[3] Kakogawa J, Sumimoto K, Ho E, Kanayama N. Transabdominal measurement of oxygenation of the placenta by nearinfrared spectroscopy. Semin Thromb Hemost. 2005;31:297301.

[4] Kaufmann P, Black S, Huppertz B. Endovascular trophoblast invasion: implications for the pathogenesis of intrauterine growth retardation and preeclampsia. Biol Reprod. 2003; 69:1-7.

[5] Kawamura T, Kakogawa J, Takeuchi Y, Takani S, Kimura S, Nishiguchi T, et al. Measurement of placental oxygenation by transabdominal near-infrared spectroscopy. Am J Perinatol. 2007;24:161-6.

[6] Ogino S, Redline RW. Villous capillary lesions of the placenta: Distinctions between chorangioma, chorangiomatosis, and chorangiosis. Hum Pathol. 2000;31:945-54.

[7] Prefumo F, Sebire NJ, Thilaganathan B. Decreased endovascular trophoblast invasion in first trimester pregnancies with high-resistance uterine artery Doppler indices. Hum Reprod. 2004;19:206-9.

[8] Redline RW. Clinical and pathological umbilical cord abnormalities in fetal thrombotic vasculopathy. Hum Pathol. 2004;35:1494-8.

[9] Redman CW, Sargent IL. Latest advances in understanding preeclampsia. Science (New York, NY). 2005;308:1592-4.

[10] Suzuki S, Takasaki S, Ozaki T, Kobayashi Y. A tissue oxygenation monitor using nir spatially resolved spectroscopy. Proc SPIE. 1999;3597:582-92.

The authors stated that there are no conflicts of interest regarding the publication of this article.

Received January 9, 2009. Revised June 23, 2009. Accepted July 28, 2009. Previously published online November 24, 2009. 